\title{
A espectroscopia de fluorescência sincronizada aplicada na análise qualitativa e quantitativa de hidrocarbonetos policíclicos aromáticos em amostras d'água
}

\author{
Synchronized fluorescence spectroscopy applied in qualitative and quantitative \\ analysis of polycyclic aromatic hydrocarbons in water samples
}

Jônatam Moreira Rodrigues*1, Gustavo Ruivo Salmazzo ${ }^{1}$, Carlos Fabiano Capato ${ }^{1}$, Evaristo Alexandre Falcão ${ }^{1}$, Anderson Rodrigues Lima Caires ${ }^{1}$, Eriton Rodrigo Botero ${ }^{1}$

${ }^{1}$ Universidade Federal da Grande Dourados, Dourados, Brasil

\begin{abstract}
Resumo
Os Hidrocarbonetos Policíclicos Aromáticos (HPAs) são conhecidos por serem altamente tóxicos. Diferentes métodos são utilizados para identificação destes compostos em amostras ambientais, entretanto, quase sempre, envolvem alta complexidade para as análises e custos significativamente altos. Contudo a técnica de Espectroscopia de Fluorescência Sincronizada (EFS) se mostra altamente simples e eficaz no processo de identificação destas substâncias. Neste contexto, o trabalho objetivou realizar um estudo quali-quantitativo de HPAs presentes em matrizes aquosas no intuito de propor a técnica de EFS como uma ferramenta para o monitoramento ambiental. Após as análises estatísticas verificou-se que o limite de deteç̧ão foi abaixo de 0,7x10${ }^{9}$ g. $m L^{-1}$ para os oito HPAs identificados pela técnica de EFS, valor este recomendado pelo Ministério da Saúde no Brasil, demonstrando que a técnica é altamente sensível e aplicável a rotina de analises em amostras de água.
\end{abstract}

Palavras-chave: Fluorescência sincronizada, HPAs, amostras de água.

\begin{abstract}
Polycyclic Aromatic Hydrocarbons (PAHs) are known to then high toxicity. Different methods are used to identify these compounds in environmental samples. However, often the methods involve high complexity experiments and high costs for operations. However, the Synchronous Fluorescence Spectroscopy (SFS) shows to be a highly simple and effective experimental technique in the identification of these substances. In this context, this study aimed to conduct a qualitative and quantitative study of PAHs present in aqueous in order to propose this technique as a tool for environmental monitoring arrays. After statistical analyzes, was identified the limit of detection below 0.7 $\times 10^{-9} \mathrm{~g} \cdot \mathrm{mL} \mathrm{L}^{-1}$ for the eight PAHs identified by the SFS. This is a value recommended by the Ministry of Health in Brazil, demonstrating that the technique is highly sensitive and applicable to analysis in water samples.
\end{abstract}

Keywords: Synchronized Fluorescence, PAHs, water samples. 


\section{Introdução}

$\mathrm{D}$ entre os problemas ambientais, uma atenção especial quanto à exposição de compostos como os Hidrocarbonetos Policíclicos Aromáticos (HPAs) é necessária. Para detectar estas substâncias em qualquer tipo de ambiente é necessário não apenas os conhecimentos relacionados aos sistemas biológicos, mas também é necessário conhecer e aplicar os conhecimentos relacionados aos aspectos físico-químicos destes poluentes, como também compreender o comportamento em relação à dispersão no meio ambiente (Sang et al, 2009).

Os HPAs são resíduos provenientes da queima de combustíveis fósseis, florestas, carvão, resíduos industriais, efluentes, entre outros processos que liberam estes poluentes para o meio ambiente (Saitoh, Itoh \& Hiraide, 2009; Meire; Azeredo \& Machado, 2007). Mais de 100 tipos diferentes de HPAs já são conhecidos (Caruso \& Alaburda, 2008; Tarpani et al, 2011). Quando presente no meio ambiente, os HPAs podem envolver diferentes problemas, como a biomagnificação ou a incorporação por algum organismo, uma vez que estudos já constataram a presença de HPAs em tecidos vegetais (algas) (Okay et al, 2000), na bile de peixes (Johnson-Restrepo et al, 2008), leite materno e urina de pessoas adultas (USEPA, 2013; ATSDR, 2013).

De um modo geral, sabe-se, que quando em contato com qualquer organismo, estes poluentes podem ser causas de doenças e sintomas diversos (USEPA, 2013), porém a maior preocupação está relacionada ao efeito mutagênico e carcinogênico apresentado por estes compostos (Krupadam et al, 2009).

Sabendo-se da nocividade destas substâncias para os seres vivos, agências de prevenção à saúde e de proteção ambiental em diferentes países apresentam regulamentações quanto à concentração destas substâncias no ambiente. No Brasil, por exemplo, o Ministério da Saúde (2011), por meio da Portaria № 2914, especifica que o HPA Benzo[a]pireno não pode ser encontrado em ambientes aquáticos em concentração superior 0,7 ng. $\mathrm{ml}^{-1}$, devido seu alto potencial tóxico.

Entre as técnicas utilizadas para identificar HPAs, a Espectroscopia de Fluorescência Sincronizada (EFS) é uma técnica promissora para análise de moléculas orgânicas, devido sua simplicidade, seletividade e seu baixo custo de operação (Vo Dinh; Fetzer \& Campiglia, 1998; Eiroa et al, 2010). A técnica de fluorescência permite identificar diferentes tipos de compostos, uma vez que os processos de excitação e emissão de luz combinados, carregam as identidades de cada substância a nível molecular. (Na Zhou et al, 2009; Cai, Zhu, Zhang, 2008). No caso específico dos HPAs, para iniciar o processo de transição eletrônica é necessário que estas moléculas recebam energia com comprimentos de onda correspondente a região do ultravioleta (Patra, 2001; Meira et al, 2011). Levando em consideração esses aspectos, este estudo tem o intuito de demonstrar a viabilidade da técnica como uma ferramenta para monitoramento ambiental, especificamente na detecção de HPAs em ambientes aquáticos.

\section{Materiais e Métodos}

\subsection{Instrumentação}

As análises foram realizadas em um espectrofluorímetro Cary Eclipse (Varian). O espectrofluorímetro possui como fonte de excitação uma lâmpada pulsada de Xenônio $(80 \mathrm{~Hz})$, com a largura a meia altura do pulso de aproximadamente $2 \mu$ s e potência de pico equivalente a 75 KW. A detecção da radiação emitida é realizada por um tubo fotomultiplicador (R928). Para realização das medidas de Espectroscopia Fluorescência Sincronizada foi utilizado uma célula de quartzo de $1 \mathrm{~cm}$ de quatro faces polidas.

\subsection{Parâmetros de Análise}

No processo de análise de HPAs utilizando a Técnica de Fluorescência Sincronizada os espectros eletromagnéticos são obtidos por meio de uma configuração adequada do espectrofluorímetro. Assim, o equipamento é capaz de realizar movimentos simultâneos dos monocromadores de excitação e emissão, mantendo a diferença entre eles constante ou sistematicamente variável. Nesta técnica, a diferença constante entre os comprimentos de onda de excitação e de emissão é denominada Delta Lambda $(\Delta \lambda)$, e cada $\Delta \lambda$, é ajustado para melhor visualizar e identificar uma substância. Neste estudo, as amostras com HPAs foram excitadas com comprimentos de onda que foram de 200 a $600 \mathrm{~nm}$, com $\Delta \lambda$ ajustados em intervalo de 10 $\mathrm{nm}$. As fendas de excitação e emissão foram ajustadas em 2,5 e $5 \mathrm{~nm}$, respectivamente. A voltagem do detector manteve fixa em $700 \mathrm{~V}$.

\subsection{Solventes e Padrões}

Neste trabalho utilizou-se álcool metílico espectroscópico com 99,9\% de pureza (UV/HPLC) e água deionizada. Os padrões de HPAs utilizados foram obtidos da Sigma-Aldrich com 98\% de pureza. Entre os HPAs presentes na solução estão o Pireno, Benzo[a]pireno, Benzo[a]antraceno, Antraceno, Benzo[k]fluoranteno, Dibenzo[a,h]antraceno, Fenantreno, Criseno, Acenaftileno, Benzo[b]fluoranteno, Benzo[g,h,i]perileno, Fluoreno, Indeno[1,2,3 - c,d]pireno.

\subsection{Preparo de soluções}

Para as análises de fluorescência foi utilizada solução de álcool metílico e água na proporção de 1:1 em volume. O álcool metílico é um solvente orgânico que auxilia na solubilização dos HPAs em água, contribuindo no pro- 
cesso de análises por fluorescência. A solução trabalho composta por uma mistura de HPAs apresentou uma concentração de $1 \times 10^{-5} \mathrm{~g} \cdot \mathrm{mL}^{-1}$ para cada HPA.

\section{Resultados e Discussões}

\subsection{Análises de HPAs por fluorescência}

Sabe-se que os HPAs podem estar dispersos em todo o meio ambiente. Nos sistemas aquáticos os hidrocarbonetos apresentam um comportamento peculiar devido a sua solubilidade. No entanto, diferentes agentes podem contribuir para a dispersão destas substâncias no ambiente. Para análises técnicas, a presença de álcool metílico em solução é um importante fator no processo de estudo dos HPAs, visto que, este solvente age como um co-solvente no processo de diluição destas substâncias em soluções aquosas (Cavalcante, 2007).

Desta forma, utilizando a proporção de 1:1 entre álcool metílico e água, as análises foram realizadas por meio da Técnica de Espectroscopia de Fluorescência Sincronizada com o intuito de verificar a eficiência desta ferramenta como forma de identificação de hidrocarbonetos presente na solução, composta por multicomponentes de HPAs.

Após as análises, observou que o $\Delta \lambda$ de $40 \mathrm{~nm}$ possibilitou a identificação de HPAs como o Pireno e o Benzo[a]pireno, demonstrado em três níveis de concentração (Figura 1). De acordo com Patra \& Mishra (2001), estes compostos absorvem energia em torno de 334 e 366 nm, e emitem fluorescência máxima em 374 e $406 \mathrm{~nm}$, respectivamente, quando utilizada a Técnica de Espectroscopia de Fluorescência Sincronizada.
A seletividade da técnica está relacionada à capacidade do equipamento captar a fluorescência em pontos específicos. Quando selecionamos o $\Delta \lambda$ de $40 \mathrm{~nm}$ para identificar o Pireno (absorção máxima de energia em $334 \mathrm{~nm}$ ), a fluorescência máxima deste composto é registrada a $40 \mathrm{~nm}$ após o ponto de máxima absorção de energia, em $374 \mathrm{~nm}$. Este fator apresentado pela técnica evita a contribuição de luminescência apresentada pela fluorescência de outros HPAs.

Analisando-se ainda a solução com os padrões de HPAs, no entanto, com o $\Delta \lambda$ de $100 \mathrm{~nm}$, foi possível identificar nos espectros outros cincos HPAs, o Fenatreno, Criseno, Benzo[a]antraceno, Dibenzo[a,h]antraceno e o Benzo[k]fluoranteno (Figura 2). Os pontos de máxima absorção de energia para estes cinco HPAs foram em 247, 265, 287, 296 e 307 nm, com respectivos máximos de fluorescência em 347, 365, 387, 396 e 407 nm (Patra \& Mishra, 2001).

Já com os espectros obtidos empregando o $\Delta \lambda$ de 160 nm, apresentados na Figura 3, foi possível identificar o Antraceno. Este composto absorve energia em torno de 245 nm e emite fluorescência máxima em 405 nm (Patra \& Mishra, 2001). Este composto apresentou alta sensibilidade para a Técnica de Fluorescência Sincronizada.

Outros autores, como He; Lin \& Li (2005) também pesquisaram a presença de alguns HPAs (Benzo[a]pireno, Pireno e Perileno) em solução usando a Técnica de Fluorescência Sincronizada. Os relatos de sucesso mostram que a técnica foi eficiente e seletiva no processo de identificação e quantificação simultânea de diferentes compostos em solução aquosa, contudo, na literatura pesquisada os HPAs eram solubilizados com o uso de compostos conhecidos como surfactantes.

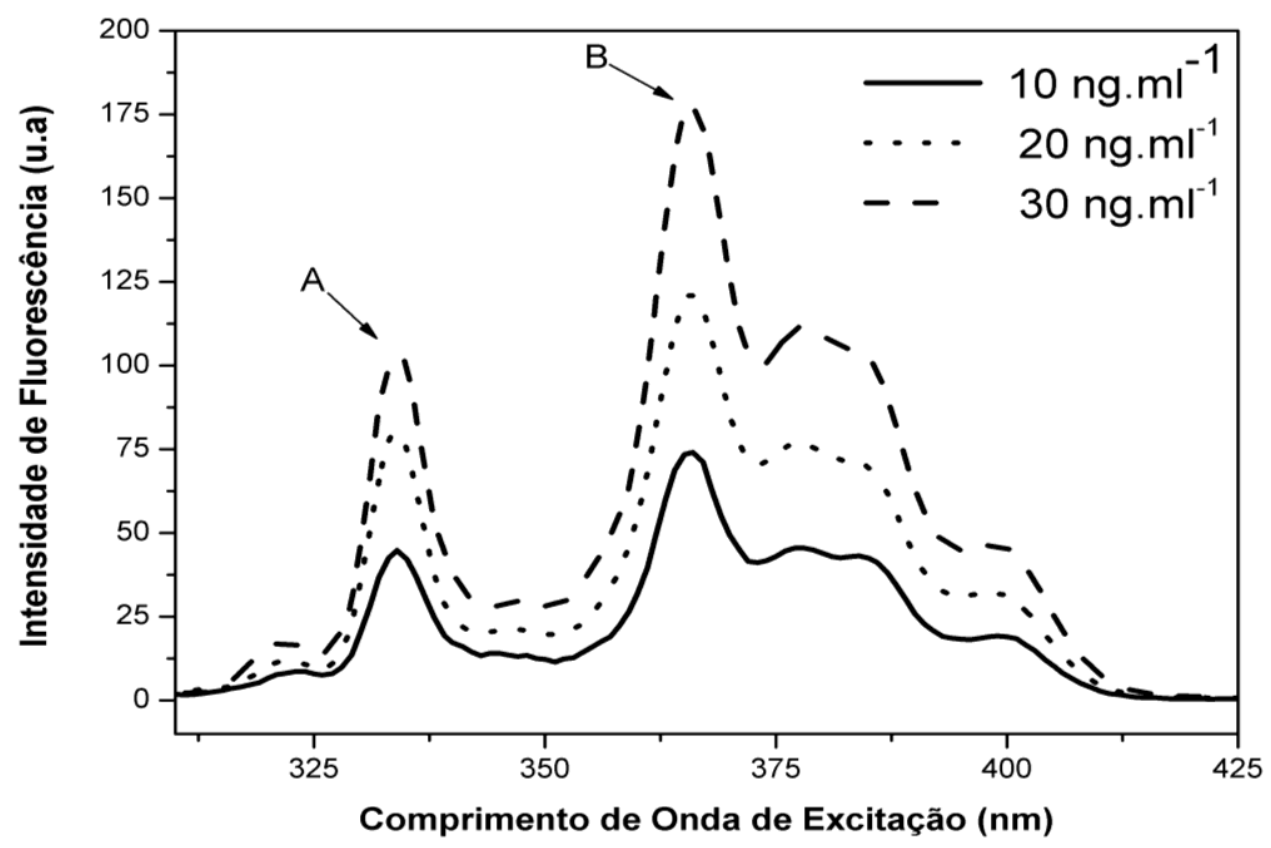

Figura 1: Espectros de excitação em função da fluorescência do Pireno (A) e do Benzo[a]pireno (B) 


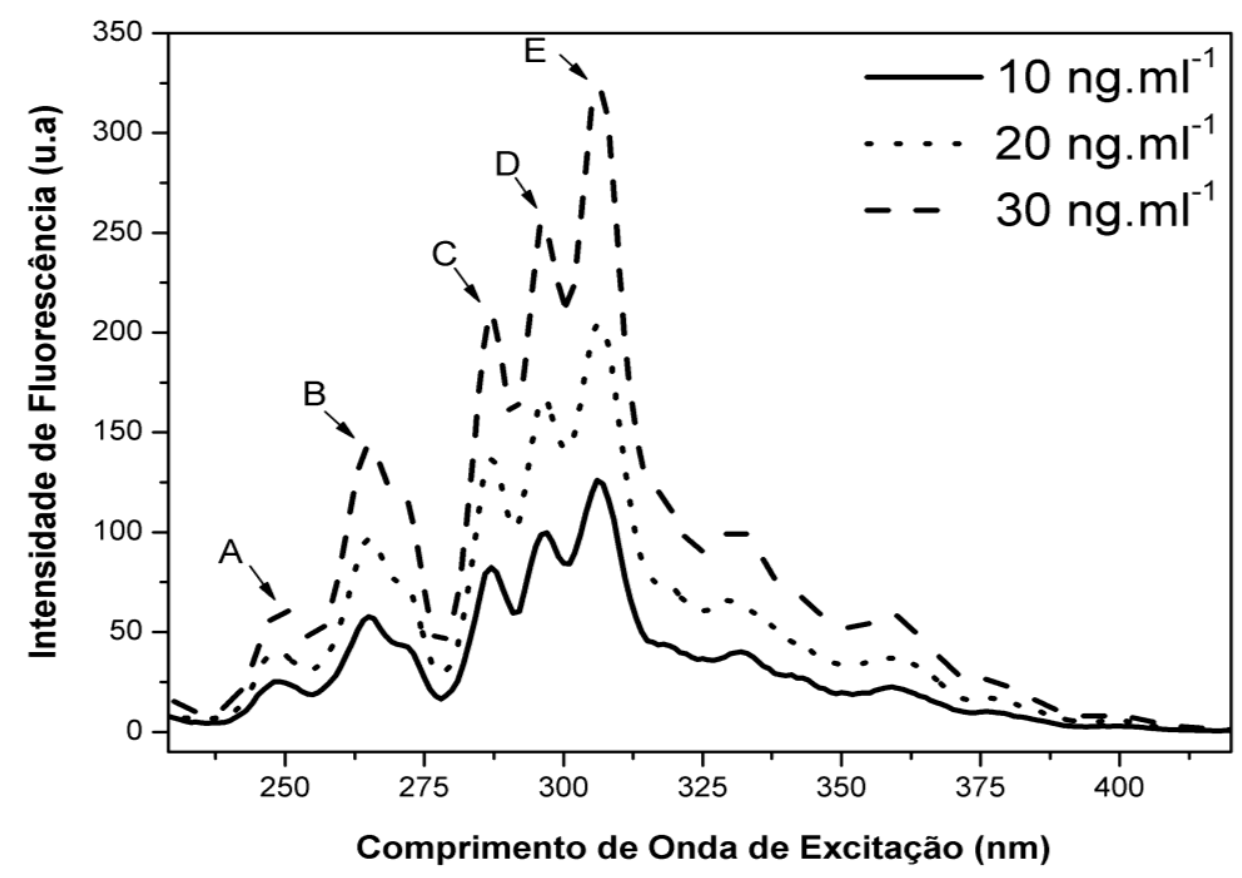

Figura 2: Espectros de excitação em função da fluorescência do Fenantreno (A), Criseno (B), Benzo[a]antraceno (C), Dibenzo[a,h]antraceno (D) e Benzo[k]fluoranteno (E).

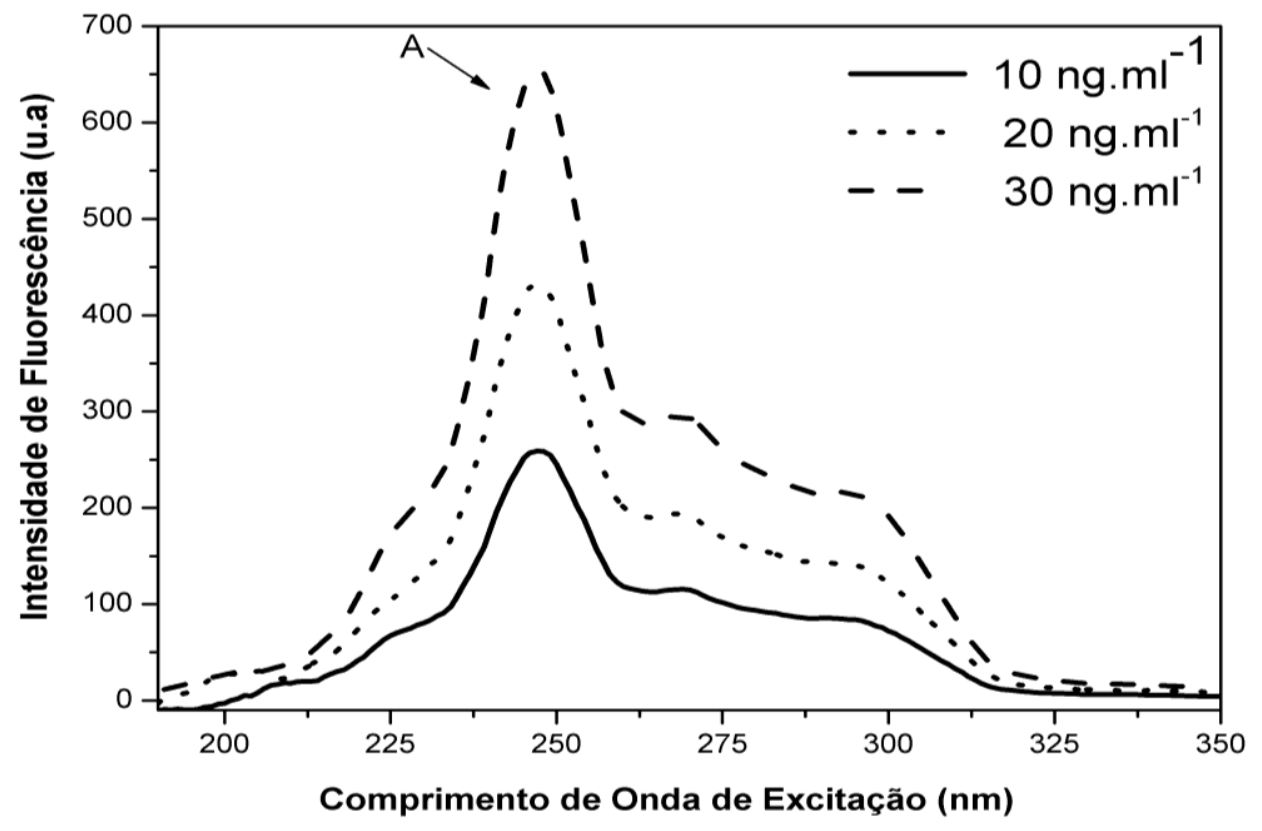

Figura 3: Espectros de excitação do Antraceno (A) em função da fluorescência

Nas amostras ambientais os HPAs são encontrados em números e tipos variáveis, no entanto, a seletividade da técnica possibilita a identificação destes compostos em meio uma complexa mistura de HPAs em rápidas análises, o que caracteriza esta técnica como uma poderosa forma de monitorar estes poluentes no meio ambiente.

Após as análises qualitativas dos oito tipos de HPAs em um meio complexo, foi possível construir uma curva de calibração para cada composto. Na Figura 4 é possível verificar a correlação entre a intensidade de fluorescência e a concentração em ng. $\mathrm{mL}^{-1}$, para cada HPA. A faixa linear foi de 2,5 a 110 ng. $\mathrm{ml}^{-1}$ na solução com os fluoróforos, como apresentado nos gráficos da Figura 4.

Seguindo as recomendações da ANVISA (2003), foram calculados os valores para o coeficiente de correlação linear (r). Os valores foram de 0,9971 para o Benzo[a] 


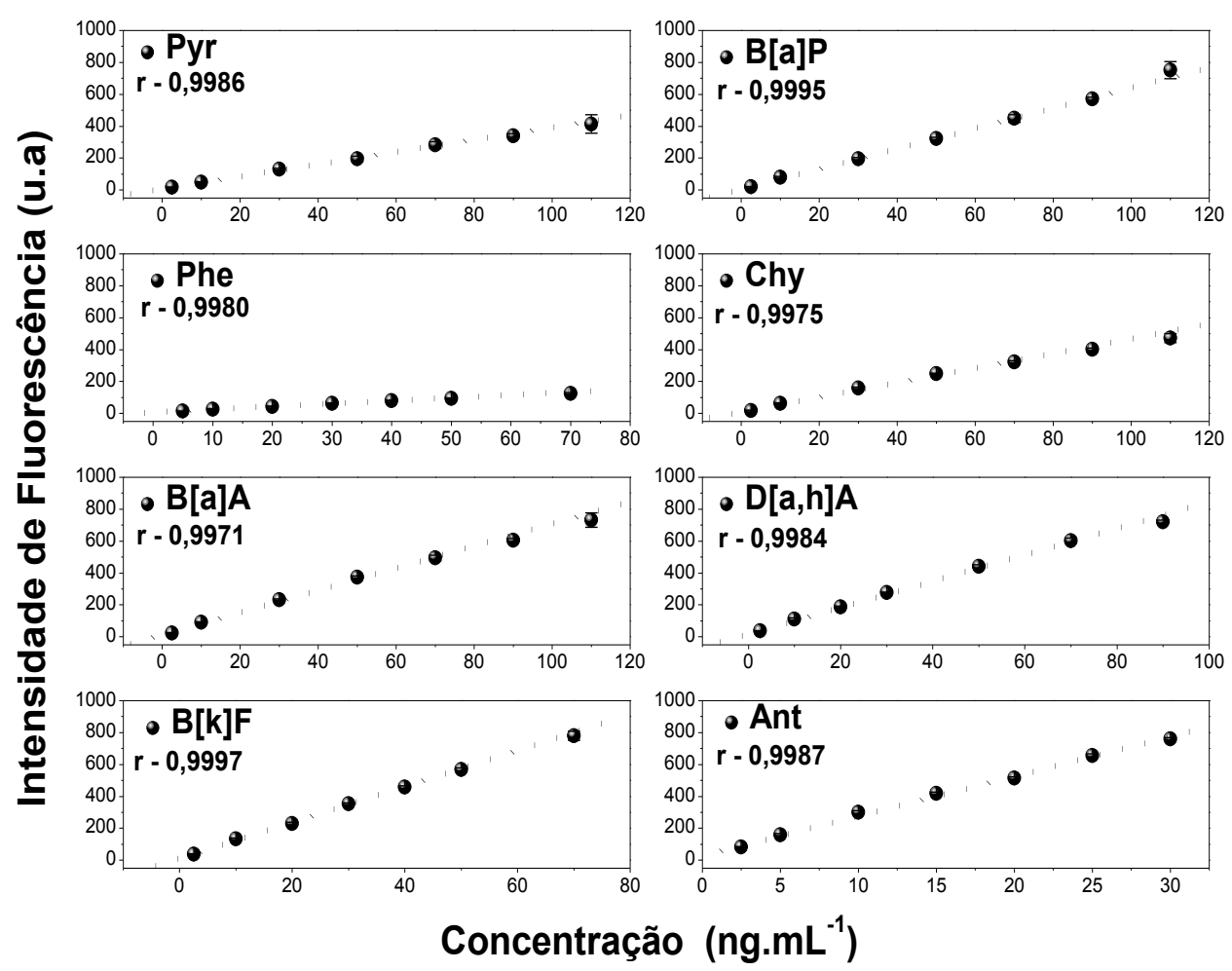

Figura 4: Curva de calibração para o Pireno (Pyr), Benzo[a]pireno (B[a]P),Fenatreno (Phe), Criseno (Chy), Benzo[a]antraceno (B[a]A), Dibenzo[a,h]antraceno(D[a,h]A), Benzo[k]fluoranteno (B[k]F) e Antraceno (Ant). Coeficiente de Correlação Linear (r).

antraceno a 0,9997 para o Benzo[k]fluoranteno. A resposta apresentada pelo método analítico foi diretamente proporcional à concentração dos analitos analisados na faixa de concentração estudada. Também foi calculado o limite mínimo de detecção para o equipamento para cada composto em solução aquosa. Percebeu-se que o Antraceno entre os HPAs foi o que apresentou maior sensibilidade para a técnica. Os valores foram obtidos em análises com soluções sem qualquer processo de préconcentração do analito (poluente), diferentemente do que é necessário para outras técnicas analíticas.

Estudos utilizando cromatografia como técnica de análise em amostras de água, obtiveram limite de detecção entre 1 a 3,5 ng.mL ${ }^{-1}$ para o Pireno, Benzo[k]fluoranteno e Benzo[a]pireno (Negrão \& Alpendurada, , 1998; Hou
\& Lee, 2002). Estes valores foram obtidos após processo de pré-concentração. Já estudos utilizando fluorescência obtiveram valores para detecção de HPAs de 0,3 a 4,1 para hidrocarbonetos como Benzo[a]pireno, Pireno e Fenantreno (Patra \& Mishra., 2001; He, Lin \& Li, 2005)

Importante ressaltar que os valores do limite de detecção apresentado neste estudo foram inferiores ao recomendado pelo Ministério da Saúde, que determina um valor limite de $0,7 \times 10^{-9} \mathrm{~g} \cdot \mathrm{mL}^{-1}$ para o Benzo[a]pireno, único HPA listado por esta agências como de alto risco a saúde. Este HPA é visto como o de maior importância devido ao alto nível de toxidade e mutagenicidade. Este HPA é utilizado como referência dentre todos os HPAs (Ministério da Saúde, 2011).

Tabela I: Valores obtidos da curva de calibração: Limite de Detecção (L.D.) e Limite de Quantificação (L.Q.) do método

\begin{tabular}{|c|c|c|c|c|c|}
\hline HPAs & $\begin{array}{c}\text { L.D. } \\
\text { (ng.ml-1) }\end{array}$ & $\begin{array}{c}\text { L.Q. } \\
\text { (ng.ml-1) }\end{array}$ & HPAs & $\begin{array}{c}\text { L.D. } \\
\text { (ng.ml-1) }\end{array}$ & $\begin{array}{c}\text { L.Q. } \\
\text { (ng.ml-1) }\end{array}$ \\
\hline Pyr & 0,55 & 1,84 & $\mathrm{D}[\mathrm{a}, \mathrm{h}] \mathrm{A}$ & 0,19 & 0,65 \\
\hline$B[a] P$ & 0,24 & 0,80 & Phe & 0,76 & 2,53 \\
\hline Chy & 0,41 & 1,35 & $\mathrm{~B}[\mathrm{k}] \mathrm{F}$ & 0,17 & 0,58 \\
\hline $\mathrm{B}[\mathbf{a}] \mathrm{A}$ & 0,21 & 0,70 & Ant & 0,07 & 0,22 \\
\hline
\end{tabular}


Considerando os aspectos principais desta técnica, podemos destacar fatores como à alta sensibilidade e capacidade para identificação de diferentes compostos em matrizes complexas sem a necessidade de algum processo para pré-concentrar os inúmeros tipos de HPAs. Outro aspecto importante está relacionado à simplicidade e agilidade para manuseio da técnica durante a realização das medidas.

\section{Conclusões}

Com base nos dados apresentados, pode-se concluir que a Técnica de Espectroscopia de Fluorescência Sincronizada é uma opção viável como ferramenta para monitoramento ambiental no intuito de determinar diferentes tipos de HPAs disseminados em ambientes aquáticos, visto sua capacidade de identificar e quantificar de forma ágil e sensível diferentes tipos de hidrocarbonetos em concentrações ínfimas, incluindo, principalmente, o Benzo[a]pireno que é exclusivamente recomendado pela Ministério da Saúde.

\section{Agradecimento}

Aos parceiros do Grupo de Óptica Aplicada (GOA) e Laboratório de Cromatografia e Espectrometria Aplicada (LECA), ambos localizados na Universidade Federal da Grande Dourados - UFGD.

\section{Referências}

\section{AGENCY FOR TOXIC SUBSTANCES}

AND DISEASE REGISTRY (ATSDR).

Case studies in environmental medicine toxicityofPolycyclicAromaticHydrocarbons (PAHs).Disponível em < http://www.atsdr.cdc.gov/ csem/csem.asp? csem $=13 \&$ po $=0>$ acesso em 15 de fevereiro de 2013.

ANVISA - Agência Nacional de Vigilância Sanitária. Guia para validação de métodos analíticos e bioanalíticos. Resolução no 899, 2003. 17p.

CAI, Z. Q.; ZHU, Y. X.; ZHANG, Y. Simultaneous determination of dissolved anthracene and pyrene in aqueous solution by synchronous fluorimetry. Spectrochimica Acta, v. 69, p. 130-133, 2008.

CARUSO, M. S. F.; ALABURDA, J. Hidrocarbonetos Policíclicos Aromáticos - Benzo[a]pireno: uma revisão. Revista. Instituto Adolfo Lutz, v. 67, p. 1-27, 2008.

CAVALCANTE, R.M. et al. Utilização da extração em fase sólida (SPE) na determinação de hidrocarbonetos policíclicos aromáticos em matrizes aquosas ambientais. Química Nova, v. 30, p. 560-564, 2007.

EIROA, A. A. et al. Critical Approach to Synchronous Spectrofluorimetry. II Trends In Analytical Chemistry, v. 29, p. 902-927, 2010.

HE, L. F.; LIN, D. L.; LI, Y. Q. Micelle-sensitized constant-energy synchronous Fluorescence spectrometry for the simultaneous determination of pyrene, Benzo[a]pyrene and Perylene. Analytical Sciences, v. 21, p. 641-645, 2005.

HOU, L.; LEE, H. K. Application of static and dynamic liquid-phase microextraction in the determination of polycyclic aromatic hydrocarbons. Journal of Chromatography A, v. 976, p. 377-385, 2002.

JOHNSON-RESTREPO, B. et al. Polycyclic Aromatic Hydrocarbons and their hydroxylated metabolites in fish bile and sediments from coastal waters of Colombia. Environmental Pollution, v. 151, p. 452459, 2008.

KRUPADAM, R. J. et al. Fluorescence spectrophotometer analysis of polycyclic aromatic hydrocarbons in environmental samples based on solid phase extraction using molecularly imprinted polymer. Environmental Science \& Technology. v. 43, p. 2871-2877, 2009.

MEIRA, M. et al. Identificação de adulteração de biocombustível por adição de óleo residual ao diesel por espectrofluorimetria total $3 \mathrm{~d}$ e análise das componentes principais. Quimica Nova, v. 34, p. 621-624, 2011.

MEIRE, R. O.; AZEREDO, A. T.; MACHADO, P. M. Aspectos ecotoxicológicos de Hidrocarbonetos Policíclicos Aromáticos. Oecologia Brasiliensis, v. 11, p. 188-201, 2007.

MINISTÉRIO DA SAÚDE. Portaria no 2.914, de 12 de dezembro de 2011. Brasília, 2011. 34p.

NA ZHOU et al. Highly sensitive and selective spectrofluorimetric approach for the rapid determination of trace benzo $[\alpha]$ pyrene in drinking water and in solutions leached from disposable paper cups. Luminescence, v. 26, p. 35-43, 2011.

NEGRÃO, M. R.; ALPENDURADA, M. F. Solventfree method for the determination of polynuclear aromatic hydrocarbons in waste water by solid- 
phase microextraction-highperformance liquid chromatography with photodiode-array detection. Journal of Chromatography A. Vol. 823, 211-218, 1998.

OKAY, O. S. et al. The role of algae (Isochrysisgalbana) enrichment on the bioaccumulation of benzo[a]pyrene and its efects on the blue mussel Mytilusedulis.Environmental Pollution, v. 110, p. 103-113, 2000.

PATRA, D.; A. K. MISHRA. Investigation on simultaneous analysis of multicomponent Polycyclic Aromatic Hydrocarbon mixtures in water samples: a simple synchronous fluorimetric method. Talanta, v. 55, p. 143-153, 2001.

SAITOH, T; ITOH, H \& HIRAIDE, M. Admicelleenhanced synchronous fluorescence spectrometry for the selective determination of polycyclic aromatic hydrocarbons in water. Talanta, v. 79, p. 177-182, 2009.

SANG, L. Z. et al. Simultaneous fluorimetric determination of the biodegradation processes of dissolved multi-component PAHs. Talanta, v. 78, p. 1339-1344, 2009.

TARPANI, L et al. Solid-phase analysis of polycyclic aromatic hydrocarbons by fluorimetric methods. Applied Spectroscopy, v. 65, p. 1342-1347, 2011.

MINISTÉRIO DA SAÚDE - Secretaria de Vigilância em Saúde. Portaria MS n. 18/2004. Série E. Legislação de Saúde, Brasília - DF, 2011.

UNITED STATES ENVIRONMENTAL PROTECTION AGENCY (USEPA).

Nationalprimarydrinkingwaterregulations. Disponível em < http://water.epa.gov/drink/ contaminants/upload/mcl-2.pdf >acesso em 21 de Janeiro de 2013.

VO DINH, T.; FETZER, J.; CAMPIGLIA, A. D. Monitoring and characterization of polyaromatic compounds in the environment. Talanta, v. 47, p. 943-969, 1998 